\title{
Algérie/France, dossier coordonné par Christopher Pinet
}

\section{Claudia Mansueto Pinet}

\section{(2) OpenEdition \\ 1 Journals}

\section{Edizione digitale}

URL: http://journals.openedition.org/studifrancesi/5796

DOI: 10.4000/studifrancesi.5796

ISSN: 2421-5856

\section{Editore}

Rosenberg \& Sellier

\section{Edizione cartacea}

Data di pubblicazione: 1 septembre 2011

Paginazione: 468-470

ISSN: 0039-2944

\section{Notizia bibliografica digitale}

Claudia Mansueto Pinet, «Algérie/France, dossier coordonné par Christopher Pinet», Studi Francesi [Online], 164 (LV | II) | 2011, online dal 30 novembre 2015, consultato il 13 janvier 2021. URL: http:// journals.openedition.org/studifrancesi/5796 ; DOI: https://doi.org/10.4000/studifrancesi.5796

Questo documento è stato generato automaticamente il 13 janvier 2021.

\section{(c) (i) (9)}

Studi Francesi è distribuita con Licenza Creative Commons Attribuzione - Non commerciale - Non opere derivate 4.0 Internazionale. 


\title{
Algérie/France, dossier coordonné par Christopher Pinet
}

\author{
Claudia Mansueto Pinet
}

\section{NOTIZIA}

Algérie/France, dossier coordonné par Christopher PINET, «The French Review», vol. 83, n. 6, May 2010, pp. 1157-1487.

1 Il numero, interamente dedicato allo stretto rapporto che lega la Francia e l'Algeria, è costituito da un'introduzione curata dallo stesso Editor (pp. 1167-1171), da una ricca sezione intitolata «Articles» (pp. 1175-1331) cui segue un nutrito corpus di recensioni, «Reviews» (pp. 1337-1410). Nell'introduzione PINET ricostruisce l'intensa relazione che lega ancora oggi la Francia all'Algeria, richiamando la necessità di riflettere sulla percezione, da parte dei Francesi, dei Maghrebini che vivono nei ghetti più sordidi delle grandi città dell'Hexagone: le tensioni, le incomprensioni, ma anche le mutue occasioni di scambio culturale e letterario tra questi due mondi non possono che risvegliare un interesse verso quella che Pinet definisce «as inextricably bound as Romeo and Juliet» (p. 1167) in cui nessuna certezza può essere assunta come dogma. Le riflessioni di Pinet, miranti a sottolineare la costante evoluzione dell'intricato processo relazionale francoalgerino, vengono riprese anche dallo scrittore Azouz BEGAG che aggiunge un'amara considerazione sul profondo disorientamento della società algerina: orientata verso una mondializzazione che sempre più spesso fa rima con banalizzazione, l'Algeria non è ancora pronta a cogliere tutte le opportunità che il progresso potrebbe affidarle. La necessità di una massiccia modernizzazione e l'incapacità di gestirla determina l'insorgenza di movimenti oltranzisti che, chiusi nell'integralismo delle proprie tradizioni islamiche, sono il segno di quanto il nuovo spaventi.

2 La ricca sezione "Articles», che si divide i alcune sotto-sezioni, contiene un «récit autobiographique» di Malika MOKEDDEM (pp. 1175-1187), seguito da un gruppo di articoli raccolti sotto il titolo «Histoire, Guerre, Camus» (pp.1189-1256) e da numerosi 
contributi sul mondo delle arti figurative riuniti nella sotto-sezione «Les Arts» (pp.1257-1331). Il récit di Malika Mokeddem si intitola L'identité traversière. Ripercorrendo la sua infanzia, gli anni della guerra e della scuola, l'autrice racconta al lettore la source di quel nomadismo congenito che la caratterizza. Legatissima alla nonna, che ha esercitato una grande influenza su di lei, Mokeddem spiega come la formazione della propria identità non passi per l'acritica acquisizione di un patrimonio culturale, ma «par le mouvement de la vie, la dynamique de la pensée» (p. 1183), perché «tout ce qu'un individu affronte dans sa vie participe de son identité» (p. 1183). Libera da costrizioni, l'autrice spiega quanto sia importante superare le frontiere, i confini, gli harem mentali come direbbe Fatima Mernissi, che imbrigliano il nostro desiderio di essere completamente déracinés, alla conquista di uno spazio interiore o fisico che ci rappresenti.

La sezione «Histoire, Guerre, Camus» è composta da cinque articoli: Camus: amitiés et question(s) algériennes (1945-1960) di Agnès SPIQUEL; Jacob's Wound: Jean Sénac, Albert Camus, and the Question of Algerian Nationalism di Katia SAINSON; Algérie, idéologie et raison: Albert Camus, Malek Bennabi, Maïssa Bey di Anne-Marie GRONHOVD; Representations of the Harkis in Lakhdar Belaid's Sérail Killers and Yasmina Khadra's La part du mort di Susan IRELAND ed infine Probing the past: Leila Sebbar, La Seine était rouge/The Seine was Red di Mildred MORTIMER.

Nel primo articolo, l'autrice cerca di chiarire la posizione di Camus nei confronti del desiderio indipendentistico dell'Algeria: in Chroniques algériennes (1957) l'intellettuale francese afferma che solo un'intensa collaborazione tra Francia ed Algeria avrebbe garantito la nascita di un nuovo stato democratico. Spiquel dimostra come le accuse mosse a Camus fossero totalmente infondate: il suo rifiuto di una guerra di indipendenza non va interpretato come disinteresse nei confronti della questione algerina, bensì come una chiara esortazione a privilegiare il dialogo. L'autrice ricorda che l'azione pacificatrice promossa da Camus coinvolse anche intellettuali algerini come Mohammed-el-Aziz Kessous che condivideva con lui la fiducia nella negoziazione per costruire una «Algérie nouvelle» (p. 1192). Spiquel ricorda che anche i più accaniti sostenitori del Fronte di Liberazione Nazionale algerino come Mouloud Feraoun e Kateb Yacine riconoscevano a Camus un reale interesse per una giusta risoluzione dei dissidi: avevano infatti ben capito, a differenza di Jean Amrouche, che preferire il dialogo alla lotta non vuol dire tradire la causa, ma contribuire alla stabilizzazione e alla crescita democratica «de cette Algérie qui souffre» (p.1194). L'articolo di Katia Sainson chiarisce la complessa relazione che univa Jean Sénac ad Albert Camus, evidenziando innanzitutto le grandi somiglianze che accomunavano i due scrittori: di origine ispanica, privati della figura paterna e cresciuti in uno stato di assoluta povertà, $i$ due intellettuali condividevano l'amore per la terra algerina e la speranza di poter vedere un giorno lo sfortunato paese maghrebino risorgere dalle ceneri di un presente deludente. Purtroppo, alla fine, come era accaduto con Amrouche, neppure Sénac comprese la straordinaria modernità del pensiero di Camus. Questi fu accusato di essere un sostenitore della "politics of clean hands» (p. 1204) e «a prisoner of his French origins» (p. 1205), indifferente di fronte al dramma degli algerini. Sainson ricorda che l'insanabile rottura tra Sénac e Camus aveva determinato nel giovane intellettuale pied-noir la nascita di un sentimento fino ad allora sconosciuto, perché il distacco dall'amato mentore lo costringe a rivivere la sofferenza patita a causa dell'abbandono del padre. Come Martinetti e come molti altri intellettuali, anche Sénac, 
alla fine del suo lungo e doloroso viaggio interiore, dovrà riconoscere che era necessario "uccidere" il padre per poter crescere. Nel terzo articolo Anne-Marie Gronhovd pone Camus al centro di un'ipotetica piramide che unisce l'intellettuale francese a Malek Bennabi e a Maïssa Bey. Convinto sostenitore del messaggio di modernità e di rinnovamento contenuto nella religione islamica, Bennabi condivide con Camus la «croyance absolue à une démocratie subséquente à l'indépendance, une Algérie hors des limites du monde soviétique ou d'un islamisme radical» (p. 1220). Purtroppo, come sottolinea la studiosa, l'insuccesso delle tesi pacifiste di Camus e Bennabi ha scaraventato l'Algeria in una spirale di corruzione, di colpi di stato e di guerre civili che ancora oggi insanguinano il paese. Citare Maïssa Bey, in qualità di pregevole interprete del clima di terrore che soffoca l'Algeria contemporanea, permette a Anne-Marie Gronhovd di riflettere su come anni di malgoverno ed di integralismo abbiano trasformato l'Algeria in un triste teatro sul quale si alternano rapaci burattini manovrati da un sistema malato, costituito da tutti quei politici e militari che «ont effacé le mot démocratie de leur vocabulaire» (p. 1228). L'articolo di Susan Ireland si sofferma sul ruolo dell'harkis, funzionario algerino alle dipendenze dello stato francese, nel corso della sanguinosa guerra d'Algeria (1954-1962). L'analisi di Sérail Killers (2000) di Lakhdar Belaïd e di La part du mort (2004) di Yasmina Khadra consente all'autrice di evidenziare come la maggior parte degli intellettuali algerini non riesca ancora a sanare le sanguinose ferite del proprio passato: $i$ tradimenti subiti, l'indifferenza francese nei confronti di una pagina storica così drammatica $\mathrm{e}$ controversa, l'odio verso il nemico sono infatti le motivazioni che spingono gli scrittori algerini a raccontare e a testimoniare affinché l'oblio non prenda il definitivo sopravvento. Per guardare con fiducia al futuro bisognerebbe tentare un comune sforzo di riconciliazione: «enterrer la hache de guerre une bonne fois pour toutes» (p. 1234) significherebbe superare vecchie tensioni e annosi risentimenti che ostacolano il processo di costruzione di un futuro condiviso. L'impossibilità di «partager l'avenir en niant le passé commun conflictuel» (p. 1242) viene espressa anche da Mildred Mortimer nel suo articolo su Leilla Sebbar che propone, nella prima parte, la rievocazione dei tragici fatti del 17 ottobre 1961 in cui centinaia di pacifici manifestanti algerini vennero uccisi dall'esercito e gettati nella Senna. L'indifferenza dei Francesi di fronte a questo drammatico evento dimostra come le piaghe di un passato taciuto e dimenticato continuino a sanguinare. Mortimer ricorda che lo scopo di Sebbar, autrice di La Seine était rouge, è proprio quello di rompere la spessa cortina di silenzio che avvolge il passato algerino, per ritrovare la forza della testimonianza, permettere ai Francesi e agli Algerini di ristabilire un dialogo visto che «none find peace in silence, even when it is their choice to remain silent» (p. 1250).

5 Recuperare una memoria storica è proprio la principale finalità di questo numero di «The French Review», dedicato all'analisi dei rapporti tra Francia ed Algeria. Tutti i contributi convergono sull'obiettivo di interpretare con imparzialità i fatti e i protagonisti dei drammatici anni della guerra algerina. Solo attraverso la rievocazione, infatti, ognuno riuscirà a creare i propri «lieux de mémoire» (p. 1251), necessari per svegliare le coscienze sopite e per smantellare definitivamente quei rassicuranti simulacri dell'amnesia che per decenni hanno impedito di fare i conti con la propria storia. 